\title{
Gonococcal conjunctivitis despite successful treatment of male urethritis
}

\section{syndrome}

Remco P.H. Peters, PhD ${ }^{1,2}$, Stephan P. Verweij, PhD ${ }^{3}$, James A. McIntyre, FRCOG ${ }^{1,4}$ and Erik

Schaftenaar, $\mathrm{MD}^{1,5,6}$

${ }^{1}$ Anova Health Institute, Johannesburg, South Africa

${ }^{2}$ Department of Medical Microbiology, University of Pretoria, South Africa

${ }^{3}$ Laboratory of Immunogenetics, Department of Medical Microbiology \& Infection Control, VU University Medical Center, Amsterdam, The Netherlands

${ }^{4}$ School of Public Health \& Family Medicine, University of Cape Town

${ }^{5}$ Rotterdam Ophthalmic Institute, Rotterdam, The Netherlands

${ }^{6}$ Department of Viroscience, Erasmus MC, Rotterdam, The Netherlands

Address for correspondence

Prof R.P.H. Peters, Anova Health Institute, 12 Sherborne Road, Johannesburg, South Africa. Tel.: +27 11 5815011. Fax: +27 11 4821116. Email: rph.peters@gmail.com.

\section{$\underline{\text { Potential conflict of interest/disclosure statement }}$}

None of the authors reports a potential conflict of interest.

\section{Funding statement.}

The Anova Health Institute is supported by the US President's Emergency Plan for AIDS Relief (PEPFAR) program via the US Agency for International Development (USAID) under Cooperative Agreement No. 674-A-12-00015. The views expressed in this manuscript do not necessarily reflect those of PEPFAR or USAID.

\section{ABSTRACT (Max 50 words)}

We report a case of progressive, cephalosporin-susceptible, Neisseria gonorrhoeae conjunctivitis despite successful treatment of male urethritis syndrome. We hypothesize that conjunctival infection progressed due to insufficient penetration of cefixime and azithromycin and point out that extra-genital infection and male urethritis may not be cured simultaneously in settings where the syndromic approach is used. 


\section{Note/case report}

A young man presented at the ophthalmology outpatient department of a hospital in rural South Africa with symptoms suggestive of conjunctivitis. He complained of progressive redness and swelling of both eyes, ocular discharge and mild pain for eleven days. Eight days before presentation at the hospital, he had consulted with a local private practitioner for penile discharge and dysuria. He was treated for male urethritis syndrome with azithromycin (1g stat dose), cefixime (400mg stat dose) and metronidazole ( $2 \mathrm{~g}$ stat dose) as verified with his private practitioner. His genital symptoms had resolved following this antibiotic treatment, but ocular symptoms progressed over time. Reliable details of sexual history could not be obtained from this patient, except for currently having a female sexual partner. Written informed consent including for ocular photography was obtained from the patient.

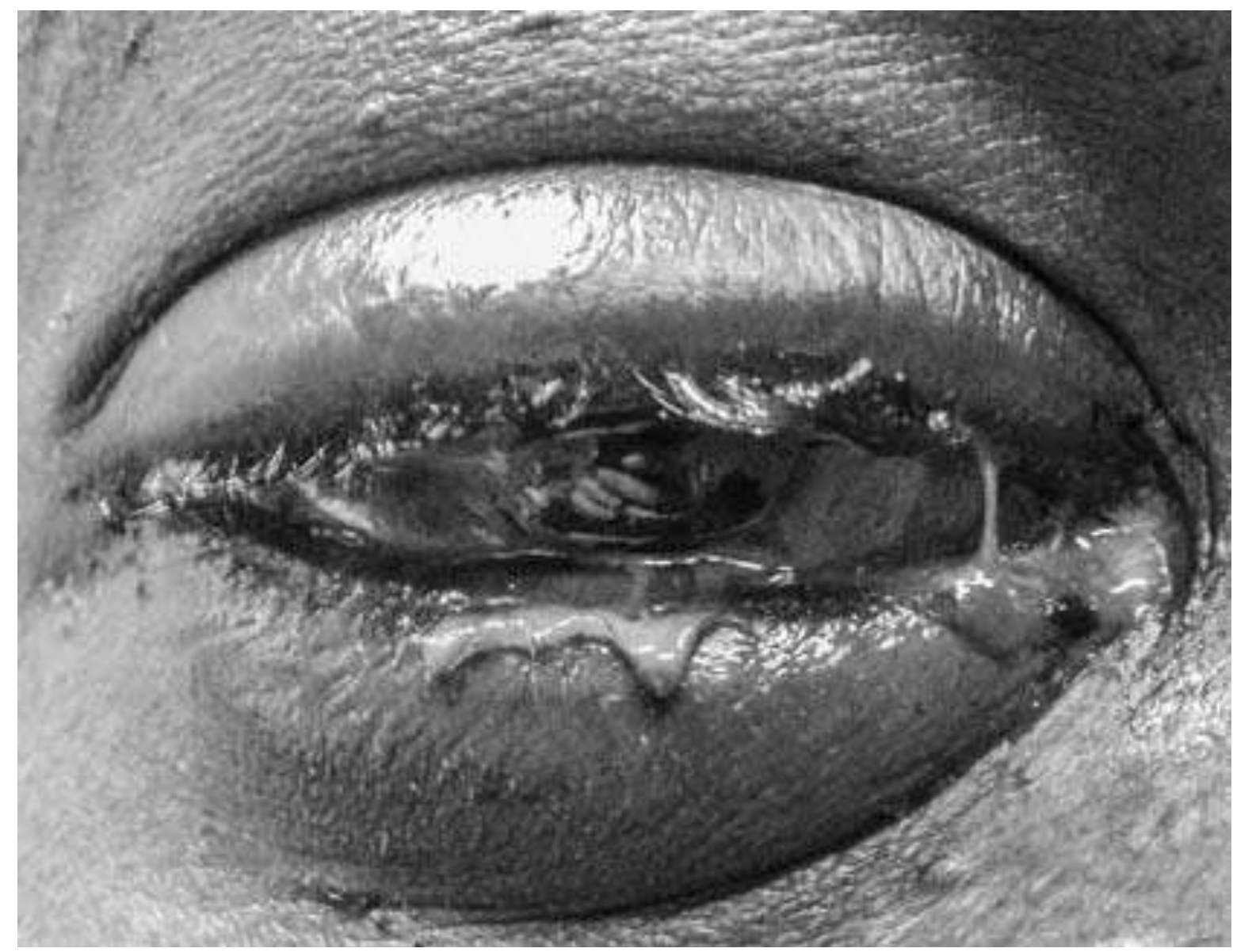

Figure 1. Mucopurulent conjunctivitis of the right eye caused by Neisseria gonorrhoeae infection 
On ocular inspection, bilateral diffuse conjunctival injection with oedema of upper- and lower eye lids was observed with profuse mucopurulent discharge (Fig 1). Slit-lamp examination did not reveal corneal abnormalities; fundoscopy was normal. Genital examination did not show any abnormalities, in particular no genital discharge was observed. The HIV rapid test was reactive and subsequent CD4 count was 205 cells $/ \mathrm{mm}^{3}$. A swab of conjunctival pus was taken for general bacterial culture and culture specific for Neisseria gonorrhoeae at the Lancet Laboratories in Tzaneen, South Africa. A second swab was obtained for PCR testing for Chlamydia trachomatis and N. gonorrhoeae. Regular rinsing of the eye was advised and, based on clinical presentation of hyperpurulent conjunctivitis, treatment for suspected uncomplicated gonococcal conjunctivitis was initiated: 1 gram of ceftriaxone intramuscular injection combined with topical ciprofloxacin $(3 \mathrm{mg} / \mathrm{mL})$ ophthalmic drops and chloramphenicol ophthalmic ointment for during the night. Ciprofloxacin drops were given as gentamicin and chloramphenicol ophthalmic drops, as indicated per South African national guidelines, were unavailable at the hospital at that time. At telephonic follow-up, the patient reported complete resolution of ocular symptoms including restoration of vision.

Microbial culture of conjunctival pus on Thayer-Martin agar revealed growth of $N$. gonorrhoeae; no other bacteria were cultivated. The antimicrobial resistance profile was as follows: penicillin susceptible (MIC of $0.016 \mu \mathrm{g} / \mathrm{mL}$ ) and, based on disk diffusion, susceptibility to cefixime and ceftriaxone. Disk diffusion tests further revealed antimicrobial resistance to ciprofloxacin and tetracycline. Unfortunately the strain was not available for further molecular characterisation due to operational logistics. PCR testing of conjunctival pus by the Abbott RealTime CT/NG Assay (Abbott Molecular, Des Plaines, IL, USA) was positive for $N$. gonorrhoeae whereas $C$. trachomatis coinfection was not detected.

To our knowledge, we report the first case of possible progression of ocular, cephalosporinsusceptible, $N$. gonorrhoeae infection after successful syndromic treatment (including cefixime and azithromycin) for male urethritis syndrome. In contrast to several recently reported cases of progressive ocular infection due to $N$. gonorrhoeae strains with reduced cephalosporin susceptibility 
or resistance, the strain isolated from the conjunctiva of our patient was fully susceptible to cephalosporins. ${ }^{1,2}$

Ocular gonorrhoea, as is the case in this patient, generally presents with eyelid swelling, conjunctival chemosis and hyperacute purulent discharge. If left untreated, the infection will progress from the conjunctiva into the cornea resulting in peripheral corneal ulceration that may rapidly progress to corneal perforation. ${ }^{3,4}$ As such, delayed initiation of antimicrobial treatment may result in significant visual impairment and, ultimately, blindness due to cornea destruction. ${ }^{4}$ In our patient, infection was limited to the conjunctiva and reported visual outcome was good after treatment.

The case presented here shows that ocular $N$. gonorrhoeae infection may persist and could progress following successful treatment of urethral discharge syndrome, including two drugs active against gonorrhoea. A limitation of this case is that we were not able to determine the cause of genital symptoms in this patient (these had resolved upon treatment and before presentation to the ophthalmology outpatient department), but the combination of drugs empirically given for urethritis syndrome would likely have cured genital $N$. gonorrhoeae infection, if present. The incubation period for ocular gonorrhoea is $3-19$ days. ${ }^{5,6}$ It is certainly not unusual for genital symptoms to precede ocular manifestations for a period of up to two weeks, in this case the ocular condition followed onset of genital symptoms by three days. Ocular symptoms were already present before treatment of urethritis syndrome was administered and showed a progressive course of disease. This makes (re)infection of the eye after exposure to treatment less likely.

Antimicrobial resistance determination showed susceptibility of the ocular N. gonorrhoeae strain to cephalosporins; unfortunately susceptibility to azithromycin could not be determined. In addition, there was resolution of genital symptoms after syndromic treatment including drugs active against N. gonorrhoeae. As such, we hypothesize that progression of ocular N. gonorrhoeae infection is not due to reduced antimicrobial susceptibility, but rather the result of insufficient penetration of these drugs in the conjunctiva to achieve cure. Tissue penetration of cefixime and subsequent drug level is relatively good in the urogenital tracts achieving cure rates of more than $97 \% .^{7}$ However, 
penetration of cefixime in extra-genital body sites is considerably lower as reflected by the cure rate of $92 \%$ and lower reported for oropharyngeal $N$. gonorrhoeae infections. ${ }^{7,8}$ To our knowledge, there are no data on cefixime and azithromycin drug levels in the conjunctiva, but ocular tissue levels of two cephalosporins, cefotaxim and cefepime, have been shown insufficient to achieve therapeutic effect for non-STI ocular infections. ${ }^{9,10}$ As such, it seems plausible that low penetration of cefixime and azithromycin in the conjunctiva resulted in lack of cure in this patient. We do not know whether the patient's HIV-infection and low CD4 cell count contributed to the course of disease.

South Africa has implemented a syndromic approach to STI management, i.e. individuals are treated empirically based on presenting symptoms and without diagnostic testing. ${ }^{11,12}$ This patient was initially treated by a private practitioner with azithromycin, cefixime and metronidazole. This combination of antibiotics would generally be adequate for urethritis syndrome, but is not in line with the recent (2015) South African sexually transmitted infections (STI) management guidelines that indicate ceftriaxone $250 \mathrm{mg}$ intramuscular injection instead of oral cefixime. ${ }^{11}$ Moreover, cefixime is not indicated for treatment of gonococcal ophthalmia in South Africa. We do not know the reason why the conjunctivitis was treated inappropriately by the private practitioner at the initial presentation. In our experience, however, cefixime use is still common in South Africa as this was the drug of choice (combined with doxycycline instead of azithromycin) in the previous version of national STI management guidelines. ${ }^{12}$ Also, prescription of oral drugs is preferred over administration of an injection by some healthcare workers and drug availability regularly plays a role in choice of antibiotics given in our setting. As such, it may be expected that cefixime will continue to be used in our setting and possibly in other resource-constrained settings.

There are limited data available to support best practice in management of ocular gonorrhoea. Based on a study by Haimovici and colleagues, who showed clinical response of ocular $N$. gonorrhoeae infection to 1 gram of ceftriaxone in 12 patients, the CDC recommends to administer 1 gram of ceftriaxone for ocular gonococcal infection, combined with 1 gram of azithromycin. ${ }^{13,14}$ There is currently no consensus about the effect of supplementing ceftriaxone injection with topical 
antimicrobial treatment on ocular N. gonorrhoeae infection. Another limitation is that the MIC for azithromycin to determine susceptibility of the $N$. gonorrhoeae strain to this drug could not be determined in our setting.

Gonococcal conjunctivitis is a condition with serious visual impact when left untreated and corneal ulceration occurs. Oral dual therapy of cefixime and azithromycin issued for male urethritis syndrome, or genital gonorrhea specifically, may fail to cure simultaneous ocular N. gonorrhoeae infection in the absence of drug resistance. This is possibly due to poor penetration of these drugs in the conjunctiva similar to other extra-genital tissues. As such, awareness of $N$. gonorrhoeae as cause of progressive conjunctivitis, as well as progression of other extra-genital infections, after syndromic treatment with cefixime and azithromycin is still warranted.

\section{REFERENCES}

1. Suzuki T, Kitagawa Y, Maruyama Y et al. Conjunctivitis caused by Neisseria gonorrhoeae isolates with reduced cephalosporin susceptibility and multidrug resistance. J Clin Microbiol 2013; $51: 4246-8$

2. Tappe D, Mueller A, Weissbrich BA, et al. Severe conjunctivitis due to multidrug-resistant Neisseria gonorrhoeae and adenovirus 53 coinfection in a traveler returning from Thailand. J Travel Med 2013; 20:403-6.

3. Wan WL, Farkas GC, May WN, et al. The clinical characteristics and course of adult gonococcal conjunctivitis. Am J Ophthalmol 1986; 102:575-83.

4. McElnea $E$, Stapleton $P$, Khan $S$, et al. Challenges in the management of Neisseria gonorrhoeae keratitis. Int Ophthalmol 2014; [Epub ahead of print].

5. Kestelyn P, Bogaerts J, Stevens AM, et al. Treatment of adult gonococcal conjunctivitis with oralnorfloxacin. Am J Ophthalmol 1989; 108:516-23.

6. Lee JS, Choi HY, Lee JE, et al. Gonococcal keratoconjunctivits in adults. Eye 2002; 16:646-9.

7. Newman LM, Moran JS, Workowski KA. Update on the management of gonorrhea in adults in the United States. Clin Infect Dis 2007; 44:S84-101.

8. Moran J. Gonorrhoea. Clin Evid 2006; 15:2162-8. 
9. Kraemer HJ, Gehrke R, Breithaupt A, et al. Simultaneous quantification of cefotaxime, desacetylcefotaxime, ofloxacine and ciprofloxacine in ocular aqueous humor and in plasma by high-performance liquid chromatography. J Chromatogr B Biomed Sci Appl 1997; 700:147-53.

10. Ozdamar A, Aras C, Ozturk R, et al. Ocular penetration of cefepime following systemic administration in humans. Ophthalmic Surg Lasers 2001; 32:25-9.

11. National Department of Health. Sexually transmitted infections - Management guidelines 2015. Adapted from: standard treatment guidelines and essential drugs list PHC. Pretoria: National Department of Health, 2015: 1-26.

12. National Department of Health. First line comprehensive management and control of sexually transmitted infections (STIS): protocol for the management of a person with a sexually transmitted infection according to the Essential Drug List. Pretoria: National Department of Health, 2008: 1-18

13. Haimovici R, Roussel TJ. Treatment of gonococcal conjunctivitis with single-dose intramuscular ceftriaxone. Am J Ophthalmol 1989, 107:511-4.

14. Workowski KA, Bolan GA, Centers for Disease Control and Prevention. Sexually transmitted diseases treatment guidelines, 2015. MMWR Recomm Rep 2015; 64:1-137. 